\title{
Commentary
}

\section{Parasites as scouts in behaviour research}

\author{
Lucie H. Salwiczek and Wolfgang Wickler
}

\begin{abstract}
L.H. Salwiczek (Iss30@cam.ac.uk), Dept. of Experimental Psychology, University of Cambridge, Downing Street, Cambridge, United Kingdom, CB2 3EB; Sub-dept. of Animal Behaviour, University of Cambridge, High Street, Madingley, Cambridge, United Kingdom CB3 8AA
\end{abstract}

W. Wolfgang (wickler@orn.mpg.de), Max Planck Institute for Ornithology, Von-der-Tann-Strasse 3, 82346 Andechs, Germany

\begin{abstract}
No living organism seems left untroubled by parasites. Parasitologists study with increasing enthusiasm when and how parasites alter host behaviour. Elucidating the neurological, endocrinological and molecular mechanisms mediating possible changes in the host's behaviour is unlikely to explain how parasites induce forms of "bizarre", "odd" or "drastically new and strange behaviours”. We argue that parasites make use of behaviour programs that already existed in their hosts rather than creating host behaviour de novo. From an evolutionary and/or ontogenetic point of view, parasites might 'fake' an everyday life signal, activate a silent sub-repertoire, or even free dormant (i.e. phylogenetically old) behaviours. We illustrate by means of a few, well-known phenomena how a thorough ethological approach will be essential in determining the origin of the shown host behaviour and to differentiate between superficially similar but separate behaviour syndromes. Parasites may even merit the label of scouts in behaviour research, unmasking behavioural capabilities not at hand in the host's standard repertoire, and occasionally they might be used as tools to draw attention to relevant nervous control areas.
\end{abstract}

Keywords: ethology, parasite, influence, host behaviour, summit disease, silent behaviour

\section{Introduction}

No animal seems left untroubled by parasites. When harbouring a parasite, an animal may fall ill or be debilitated in one way or another. In the last three decades, researchers from several disciplines have produced a flurry of publications devoted to the parasites' life cycles and their influence on host physiology and morphology. By definition, the interests of parasite and host diverge. Most recently, special attention has focused on parasites' often subtle 'strategies' to manipulate the host behaviour to their own advantage, acting indirectly and/or directly on the host's nervous system (e.g. Biron et al. 2005, Adamo 2002). Hosts may then produce - from a commonsense point of view - "bizarre” (Dawkins 1990: S66), "novel” (Biron et al. 2005: 2124), “odd” and "drastically new and strange behaviours” (Poulin 1995: 1371). Most scientists are, however, at a loss to explain the host's behaviour which then is supposedly 'created' by the parasite.

We agree with Dawkins (1982) that the parasite's genes have (extended) phenotypic expression in the host's behaviour. But we disagree on the detail that they should be able to initiate a seemingly goal-directed novel behaviour that is totally absent from the host's inherited repertoire. Instead the seemingly new and aberrant behaviour will, like any evolutionary novelty, turn out to be a modification of something already existing. 
Even mere side-effects of the infection may increase the parasite's transmission probability. But many parasites benefit from more specific host reactions that may be called either "simple" or "complex", depending upon the number and/or diversity of units (or elements) of the host's phenotype (e.g. behaviour) supposedly involved. This however does not coincide with a "simple" or "complex" manipulation strategy of the parasite which in turn might be taken as indicating the degree of a parasite's evolutionary adaptation. As we will show, simple manipulations by a parasite may cause rather complex alterations in a host's behaviour.

Our working hypothesis is that parasites do not induce "novel" behaviours but make use of already existing behaviour programs in their hosts, as LoosFrank and Zimmermann (1976: 288) put it (our translation): "that in the course of evolution parasites have taken advantage of certain host-specific schemes of behaviour". With careful consideration of the ecology and the total behavioural repertoire of a host species and its closest relatives, one should be able to identify the behaviour domain of the host which has been manipulated by the parasite. A few selected examples of recently discussed parasite-induced behaviour modifications will substantiate our point of view.

\section{Exploiting normal oviposition behaviour}

The ciliate protozoan Lambornella clarki enters its host, the female treehole-breeding Aedes sierrensis mosquito, castrates it and reproduces in the reproductive tract. The ovaries, filled not with mature mosquito eggs but with ciliate progeny, are finally fully distended and signal the female to return to a water-filled treehole. By performing oviposition behaviour, she thereby deposits the ciliates' offspring and actively disperses the parasite. Do these castrated females "mimic the oviposition behaviour of normal gravid females" (Egerter et al. 1986: 7338)?

Indeed, no mimicking behaviour is involved on the female mosquito's part. While depositing parasites instead of eggs (false oviposition) she neither fakes a signal nor imitates a model-behaviour. The mass of ciliate progeny however fakes the signal of a mass of eggs that elicits the female's normal oviposition behaviour.

\section{Activating silent sex-specific behaviour}

The aquatic larva of the mermithid nematode Gasteromermis viridis upon contact with a nymph of the mayfly Baetis bicaudatus enters it through a hole made in the host's cuticle and, absorbing nutrients from the host hemocoel, matures while the host completes its nymphal development. A female Baetis that has moulted into an adult will finally return to a river, flying upstream, and oviposit on the submerged side of a protruding rock. If she hosted a mermithid, it will on this occasion escape through a puncture wound in her abdomen (Vance 1996).

Uninfected adult males form swarms where mating occurs; they do not return to a river after their final moult. Mermithid-infected males however undertake the typical female upstream flight pattern and adopt "mock oviposition behaviour" on the side of a rock (Vance 1996: 910), allowing the parasite to escape and complete its life cycle. All those genetic males which are nevertheless found ovipositing are morphologically either intersexes or truly female in appearance (Vance 1996).

Males and females share most of their genomes. "Phenotype determination, as in "sex determination" or "caste determination" is the choice made at a decision point” (West-Eberhard 2003: 68). 'Decision' means sex-limited expression of genomic information present in both sexes, while unexpressed information remains available but falls silent. The mermithid parasite induces the expression of the female-specific behaviour subrepertoire (i.e. oviposition) in genetic male mayflies.

\section{Generating the puzzling summit disease}

Various insects, after parasite infection, climb to elevated sites, mainly on vegetation. This common behaviour has been termed Wipfelkrankheit, tree-top disease, topping behaviour or summit disease (Marikovsky 1962, Schmid-Hempel 1998). We shall exemplify it with Camponotus and Formica ants, parasitized by two liver fluke species (Dendrocoelium hospes, D. dendriticum) and a fungus (Entomophthora ovispora) respectively.

(1) The African liver fluke Dendrocoelium hospes completes its life cycle in goats, buffalos, and presumably some rodent species as its final hosts, causing dicrocoelosis (Lucius et al. 1980). Fluke eggs drop from the mammal with the feces. Limicolaria snails may also consume some fluke eggs when eating the feces. Miracidia stages hatch from the eggs, and develop into sporocycsts, which in turn generate the fluke's cercariae larvae. The snail will eventually emit great numbers of these cercariae in mucus balls. Camponotus carpenter ants, upon finding a mucus ball, readily feed from it and thus ingest fluke cercariae as well. The cercariae, distributed in the body of their second intermediate host, the ant, develop into cysts ready to infect another one of its final hosts. How do they get there?

Infected Camponotus ants suffering from so-called summit disease assemble in groups on plants and rest there day and night until they are swallowed by a grazing mammal. Most importantly, infected ants do not use their mandibles to fix themselves on the plant. Two 
cercariae, settling in the dorsal part of each antennal lobe of the deutocerebrum (Romig et al. 1980), shift the behaviour such that infected Camponotus ants behave like members of a temporal soldier caste prepared to defend the nest. While in this role, uninfected colony nest mates respond to the modified behaviour of their parasite-infested colony mates in the proper way: they feed them (Hölldobler and Wilson 1990). It seems most likely that $D$. hospes elicits a caste-specific "soldier behaviour" in Camponotus ants, thereby silencing any other caste-specific behaviour.

(2) The European liver fluke $D$. dendriticum lives in the bile ducts of the liver of cattle and sheep (and other ungulates). Eggs pass from the host with feces and are consumed by Zebrina, Helicella or Cochlicops land snails eating the feces. Again, miracidia larvae hatch from fluke eggs, develop via sporocycsts into cercariae larvae, and are eventually emitted by the snail in mucus balls. Wood-ant workers (Formica fusca, F. rufibarbis, $F$. gagates) finding a mucus ball will feed from it and thereby ingest the fluke's cercaria larvae. Within the ant, all the eaten cercariae aim towards the head, but only the first to arrive there will settle in a specific depression in the anterior part of the ant's subesophageal ganglion which is in close contact to the origin of the mandible nerves. The other cercariae develop into cysts at various places in the ant's body.

The one cercaria in the ant's head turns into a noninfective "brain-worm". It manipulates the behaviour of the worker-ant to the effect that the ant no longer returns to the nest when the temperature drops in late afternoon, but instead climbs to the top of a grass blade and spends the night there, anchored by its mandibles. Thus exposed, an infected ant is likely to be consumed up by a grazing ungulate, the fluke's definite host. If not swallowed by a grazing mammal, the ant resumes normal behaviour as temperature rises during the day; but it returns to an elevated position the next evening.

According to Dawkins (1990: S65), Formica ants on the top of a grass stem remain immobile "as if asleep". But, in fact, they do sleep! The ants demonstrate "a phylogenetically old sleeping behaviour typical for some non-social hymenoptera" (Wickler 1976: 212). Various solitary hymenoptera typically crawl up twigs, go over the top and then clasp the twig at or near its tip with their mandibles for sleep (Kaiser 1995). Linnaeus even named a bee species, Apis florisomnis, in reference to this kind of sleeping roost. Consequently, the brainworm obviously revives a common hymenopteran sleeping behaviour that in subterranean ants normally lies dormant.

(3) A Formica rufa worker-ant infected with the fungus Entomophthora ovispora seems to free the same dormant hymenopteran sleeping behaviour, inducing infected Formica rufa worker-ants to climb grasses in the evening (Loos-Frank and Zimmermann 1976); they never do so otherwise (Marikovsky 1962). Within the ant, the density of hyphae is largest in the vicinity of sub-esophageal and protocerebral nerve ganglia (LoosFrank and Zimmermann 1976). The diseased ant fastens itself irreversibly with its mandibles to the surface and usually dies once fastened to the grass blade. Some individuals are even killed by the fungus and 'glued' to the grass stem by fungal holdfasts (Roy et al. 2006) while still on their way up (Loos-Frank and Zimmermann 1976).

\section{Discussion}

We maintain that no parasite can create a truly novel behaviour in its host that was totally absent from the host's original repertoire. Instead, parasites can, to their own advantage, induce complex and spectacular changes in host behaviour by motivating their host to perform a particular behaviour in a context that is deleterious to the host. To achieve this, the parasite may manipulate key stimuli for the host's normal behaviour (as in female mosquitoes), or may elicit an inverted sex behaviour (as in male mayflies), or may evoke an inappropriate caste-specific behaviour (in Camponotus ants), or resuscitate a "dormant" behaviour that had been inactivated during evolution (as in the Formica ants). We propose that this will pertain to any parasiteinduced behaviour of any host. Having not been supplemented with any truly novel behaviour, but having one of its own (even higher-level) behaviours misused by the parasite, makes it particularly difficult for the host to develop a counter-adaptation to block this behaviour.

From an ethological perspective "summit-disease" unfolds as being the initial common element (climbing up) of different behaviours such as sleeping and standing lookout. To differentiate between those behaviours, one has to pay close attention to additional behaviour components like activity-rhythm, mandible movements (e.g. fastening to the substrate or eating respectively), or conspecifics' responses in the above cases. It does not come as a surprise, then, if phylogenetically distant parasites such as a fungus and a fluke evoke the same dormant sleeping behaviour in an infected ant, but two different flukes might elicit two different ant behaviours that share a common first component. Of course, an early death of the infected animal (as in the fungus-infected ant) may prevent the full behaviour performance required for analysis.

An ethological analysis of parasite-triggered behaviour will help to rule out a coincidental pathological consequence of the infection. It will also help to avoid unfitting interpretations of such behaviour. For example, Dicrocoelium makes Formica ants seek elevated places, use their mandibles to fasten themselves, and follow a rhythm of moving upward in 
the evening and downward in the morning; this may look like manipulating the host in more than one dimension (Cécilly and Perrot-Minnot 2005). In fact, all the parasite has to do is to evoke an original sleeping behaviour which comprises all of the behavioural components observed.

Alternatively, "if elevation seeking was initially a febrile behaviour that has been captured by some parasite" (Moore 2002: 57), then 'summit disease" could have its origin in feverishness. Some insects can defeat a parasite when they ascend vegetation and become feverish by solar radiation. Since the ants seek elevated places at night, the Formica brain-worm will additionally have to shift the ant's chronobiology. This timing shift is counter-adaptive because it wastes day time when the final host (herbivores) is grazing. We think the ethological approach arrives at the simplest explanation for the observed parasite-host interaction and facilitates the explanation of these maladaptive traits.

"Most of the mechanisms by which parasites alter behaviour remain a mystery, and the evolutionary events that enabled them to seize upon these mechanisms are equally unknown to us" (Moore 2002: 119). The most promising solution to this worrying situation, we suggest, involves a methodical analysis of all behaviour patterns available to the host, with specific awareness of imaginable obscured or dormant behaviour elements. Parasites may then even merit the label of scouts in behaviour research if they unmask behavioural capabilities not at hand in the host's standard repertoire, and occasionally even draw attention to corresponding nervous control areas.

\section{Aknowledgement}

We thank Chris Jiggins for comments on the manuscript.

\section{Referees}

Frank Cézilly - frank.cezilly@u-bourgogne.fr University de Bourgogne, France.

Otto Seppälä - otto.seppaelae@eawag.ch

ETH-Zurich, Switzerland.

\section{References}

Adamo, S.A. 2002. Modulating the modulators: parasites, neuromodulators and hostbehavioral changes. Brain Behavior and Evolution 60: 370377. CrossRef

Biron, D.G., Marché, L., Ponton, F., Loxdale, H. D., Galéotti, N., Renault, L., Joly, C. and F. Thomas. 2005. Behavioural manipulation in a grasshopper harbouring hairworm: a proteomics approach. Proceedings of the Royal Society of London 272: 2117-2126. CrossRef

Cézilly, F. and M.-J. Perrot-Minnot. 2005. Studying adaptive changes in the behaviour of infected hosts: a long and winding road. Behavioural Processes 68: 223-228. CrossRef

Dawkins, R. 1982. The extended phenotype: the gene as the unit of selection. W. H. Freeman, New York.

Dawkins, R. 1990. Parasites, desiderata lists and the paradox of the organism. Parasitology 100: S63S73.

Egerter, D. E., Anderson, J. R. and J. O. Washburn. 1986. Dispersal of the parasitic ciliate Lambornella clarki: implications for ciliates in the biological control of mosquitoes. Proceedings of the

National Academy of Sciences (USA) 83: 73357339. CrossRef

Hölldobler, B. and E. O. Wilson. 1990. The Ants. Harvard University Press, Cambridge.

Kaiser, W. 1995. Rest at night in some solitary bees - a comparison with the sleep-like state of honey bees. Apidologie 26: 213-230. CrossRef

Loos-Frank, B. and G. Zimmermann. 1976. Über eine dem Dicrocoelium-Befall analoge Verhaltensänderung bei Ameisen der Gattung Formica durch einen Pilz der Gattung Entomophthora. Zeitschrift für Parasitenkunde 49: 281-289. CrossRef

Lucius, R., Romig, T. and W. Frank. 1980. Camponotus compressiscapus André (Hymenoptera, Formicidae) an experimental second intermediate host of Dicrocoelium hospes Looss, 1907 (Trematodes, Dicrocoeliidae). Zeitschrift für Parasitenkunde 63: 271-275. CrossRef

Marikovsky, P.I. 1962. On some features of behaviour of the ants Formica rufa L. infected with fungus disease. Insectes Socieaux 9: 173-179. CrossRef

Moore, J. 2002. Parasites and the behavior of animals. Oxford University Press, Oxford.

Poulin, R. 1995. Phylogeny, ecology, and the richness of parasite communities in vertebrates. Ecological Monographs 65: 283-302. CrossRef

Romig, T., Lucius, R. and W. Frank. 1980. Cerebral larvae in the second intermediate host of Dicrocoelium dendriticum Rudolphi, 1819) and Dicrocoelium hospes Looss, 1907 (Trematodes, Dicrocoeliidae). Zeitschrift für Parasitenkunde 63: 277-286. CrossRef

Roy, H.E., Steinkraus, D.C., Eilenberg, J., Hajek, A.E. and J.K. Pell. 2006. Bizarre interactions and endgames: Entomopathogenic fungi and their arthropod hosts. Annual Review of Entomology 51: 331-357. CrossRef

Schmid-Hempel, P. 1998. Parasites in social insects. Princeton University Press, Princeton NJ. 
Vance, S.A. 1996. Morphological and behavioural sex reversal in mermithid-infected mayflies. Proceedings of the Royal Society of London Series B Biological Sciences 263: 907-912. CrossRef

West-Eberhard, M.J. 2003. Developmental plasticity and evolution. Oxford University Press, Oxford, New York.

Wickler, W. 1976. Evolution-oriented ethology, kin selection, and altruistic parasites. Zeitschrift für Tierpsychologie 42: 206-214.

\section{Response to referee}

We agree with Cézilly (2009) that a mechanistic analysis of host manipulation by parasites is not an alternative to the ethological approach; it is indeed part thereof. Countless researchers refer to Tinbergen's classic paper about the four possible answers to the question "Why do animals behave like they do?" (Tinbergen 1963), but many seem to miss the point that Tinbergen took for granted; that is, the biological phenomenon to be studied - in his case, always an observable behaviour - has first to be thoroughly described and preferably also documented on film (and/or tape). When Tinbergen (1963) defined ethology as "the biology of behaviour", he outlined in detail the ethological approach which was concerned with four kinds of general biological causation. In order to fully understand any behaviour, one must study (1) its underlying mechanisms and (2) its ontogenetic development, as well as (3) its functional selective consequences and (4) evolution during phylogeny. In cases of parasite-manipulated host behaviour, this amounts to answering the four questions with respect to both the particular actions of the host and the manipulative actions of the parasite. So far such complete knowledge is not available for any parasitehost-interaction.

We argue that, regarding the actions of an infected host, a parasitologist needs to know in which situations the same actions (or elements of them) are shown by uninfected members of the host species. Tinbergen argued that the distinction between mechanisms, ontogeny, function and evolution "is pragmatic rather than logical" (Tinbergen 1963: 426); thus if parasitic infection leads to reversed phototaxis in the host (for example see Cézilly 2009), a mechanism-oriented approach is necessary to identify the physiological causation ('mechanism') together with an ethological approach that analyses the behavioural and situational contexts in which either taxis, normal or reversed, shows up without a parasite. Comparisons of behaviour between infected and uninfected hosts are necessary to substantiate what researchers may mean when they use terms like "aberrant", "new" or "novel", and will provide clarity and consistency in the choice of terminology to prevent obscure debates. Cézilly (2009) feels that these terms simply stand for "not usually observed in uninfected hosts". However, a given behaviour may usually be shown just once per lifetime (and then easily overlooked), or it may be part of a normal daily routine; it may occur spontaneously or be strictly stimulus dependent. So, what does "not usually" mean?

Cézilly (2009) directs our attention to the precise meaning of "multidimensionality", which implies that several parasite-induced phenotypic alterations are independent from a mechanistic point of view. We agree that multidimensionality is important, and actually argue against multidimensionality in the Dicrocoelium case. In our article, we dispute Moore's scenario that actually suggests multidimensionality (Cézilly and PerrotMinnot 2005) as well as "several simultaneous phenotypic alterations” (Cézilly 2009) caused by infection.

Moore (2002: 57) speculates that "if elevation seeking was initially a febrile behaviour that has been captured by some parasite” , 'summit disease' could have its origin in feverishness. In the case of Dicrocoelium dendriticum this would suggest that the parasite (a) activates feverish behaviour, (b) manipulates its circadian rhythm to make the ant climb up in the evening rather than during the day, (c) blocks the normal evening-return to the colony, and finally (d) activates the ant's mandibles at a suitable moment to fasten the ant to the plant. Moreover, since a surviving ant resumes normal activity in the morning, but returns to an elevated position the next evening, the parasite must correspondingly de-activate and then re-activate its manipulating influences. Following Cézilly (2009), the parasite's manipulation of different independent behaviour domains in Moore's scenario would suggest multidimensionality. Our dormant-sleep behaviour of originally solitary hymenoptera, however, discloses that the behaviour elements are by no means all independent, and are thus not multidimensional in Cézilly's (2009) sense, nor do they represent a random sequence of simultaneously elicited behaviour sequences. This is an excellent example of how a thorough ethological approach identifies 'true' multidimensionality when studying parasites that activate components from different behaviour domains.

We are interested in parasites that specifically exploit part of the behavioural repertoire of the host, and Cézilly (2009) therefore draws our attention to Eberhard (2000). However, some of the data in this paper makes us question Eberhard's conclusion about "the larva's ability to induce specific behaviour patterns in the spider" (2000: 255). Eberhard (2000) gives a detailed description of the way that the long-jawed orb weaver Plesiometra argyra alters its web-spinning behaviour under the influence of the larva of the parasitoid 
ichneumonid wasp Hymenoepimecis sp. He notes that the spider's altered behaviour is "almost identical to the early stages of one subroutine of normal orb weaving" (2000: 255); variants of the resulting constructions confirmed "that they were modified orbs". Eberhard called the scaffold produced by the spider a "unique cocoon web", though its suggested uniqueness seems doubtful in view of the various forms it takes. It is true that the ichneumonid larva "spins its pupal cocoon hanging by a line from the cocoon web" (Eberhard 2000: 255), and the female spider for her own egg cocoons makes a comparable, though much less variable structure. However, web structures that look similar need not be constructed according to similar algorithms (Vollrath 1988). Interestingly, some "spiders from which the larvae were removed [...] slowly reverted to more normal orbs"; and Eberhard concluded that "the changes in the spider's behaviour must be induced chemically” (2000: 255).

Comparable web malformations have in fact been described for orb-weaving spiders under the influence of various drugs (Jackson 1974, Witt 2007). It would therefore be important to analyze the supposed chemical used by the ichneumonid larva, because it may turn out to be just another substance that affects the spider's web-spinning behaviour in a rather unspecific way. The ultimate question then is whether a mere superficial functional similarity (e.g. barriers to predators, Hieber 1992) connects the suspension systems for spider eggs and for pupating wasps. To answer that question requires finding out what makes the uninfected spider switch between her own orb-web and cocoon-web building. In the end this might help to differentiate between a "most finely directed alteration of behaviour" (as Eberhard claimed for the ichneumonid wasp) and host manipulation "by relatively straightforward mechanisms, for example by the modification of particular receptors"; Eberhard wrongly argues that the Dicrocoelium dendidritum brainworm provides an instance of the latter. Our article shows that an ethological approach can distinguish between parasite manipulation of particular behaviour elements and those that shift a complete behaviour into a different context.

"An important aspect of host manipulation by parasites lies in its efficiency” (Cézilly and PerrotMinnot 2005: 225). Efficiency on the side of the parasite could mean achieving increased probability of transmission with little effort. It might not matter to the parasite whether transmission is enhanced as a sideeffect of infection (e.g. reduced activity, blindness, weakened orientation, or impaired feeding), or through direct/indirect manipulation; "if it increases parasite fitness by increasing transmission probability, then it will be favored by selection” (Seppälä, comment). But it might matter how much a parasite has to invest, e.g. how many modifications it actually has to accomplish to alter a host's behaviour. Natural selection will favor parasites that 'find' the spot in its host (e.g. in the brain) where minimum manipulation elicits the whole behavioural syndrome necessary to increase the probability of transmission. Combining the ethological approach with estimating "net benefit of manipulation" (Cézilly and Perrot-Minnot 2005: 224) during the investigation of the various manipulation processes ('mechanisms') could further help to elucidate possible evolutionary pathways for a given host-parasite interaction.

Cézilly, F. 2009. Host-manipulation by parasites: towards a neuroethological approach? Ideas in Ecology and Evolution 2: 7-8. CrossRef

Cézilly, F. and M.-J. Perrot-Minnot. 2005. Studying adaptive changes in the behaviour of infected hosts: a long and winding road. Behavioural Processes 68: 223-228. CrossRef

Eberhard, W. G. 2000. Spider manipulation by a wasp larva. Nature 406: 255-256. CrossRef

Hieber, C. S. 1992. Spider cocoons and their suspension systems as barriers to generalist and specialist predators. Oecologia 91: 530-535. CrossRef

Jackson, R. R. 1974. Effects of d-amphetamine sulfate and diazepam on thread connection fine structure in a spider' s web. Journal of Arachnology 2: 37-41.

Moore, J. 2002. Parasites and the behavior of animals. Oxford University Press, Oxford.

Tinbergen, N. 1963. On aims and methods of ethology. Zeitschrift für Tierpsychologie 20: 410-433.

Vollrath, F. 1988. Untangling the spider's web. Trends in Ecology and Evolution 3: 331-335. CrossRef

Witt, P. N. 2007. Drugs alter web-building of spiders: a review and evaluation. Behavioral Science 16: 98113. $\underline{\text { CrossRef }}$ 